\title{
Universal Points in the Asymptotic Spectrum of Tensors ${ }^{*}$
}

\author{
Matthias Christandl \\ University of Copenhagen \\ Copenhagen, Denmark \\ christandl@math.ku.dk
}

\author{
Péter Vrana \\ Budapest University of \\ Technology and Economics \\ Budapest, Hungary \\ vranap@math.bme.hu
}

\author{
Jeroen Zuiddam \\ Centrum Wiskunde \& Informatica \\ Amsterdam, Netherlands \\ j.zuiddam@cwi.nl
}

\begin{abstract}
The asymptotic restriction problem for tensors $s$ and $t$ is to find the smallest $\beta \geq 0$ such that the $n$th tensor power of $t$ can be obtained from the $(\beta n+o(n))$ th tensor power of $s$ by applying linear maps to the tensor legs - this is called restriction - when $n$ goes to infinity. Applications include computing the arithmetic complexity of matrix multiplication in algebraic complexity theory, deciding the feasibility of an asymptotic transformation between pure quantum states via stochastic local operations and classical communication in quantum information theory, bounding the query complexity of certain properties in algebraic property testing, and bounding the size of combinatorial structures like tri-colored sum-free sets in additive combinatorics.

Naturally, the asymptotic restriction problem asks for obstructions (think of lower bounds in computational complexity) and constructions (think of fast matrix multiplication algorithms). Strassen showed that for obstructions it is sufficient to consider maps from $k$-tensors to nonnegative reals, that are monotone under restriction, normalised on diagonal tensors, additive under direct sum and multiplicative under tensor product, named spectral points (SFCS 1986 and J. Reine Angew. Math. 1988). Strassen introduced the support functionals, which are spectral points for oblique tensors, a strict subfamily of all tensors (J. Reine Angew. Math. 1991). On the construction side, an important work is the Coppersmith-Winograd method for tight tensors and tight sets.

We present the first nontrivial spectral points for the family of all complex tensors, named quantum functionals. Finding such universal spectral points has been an open problem for thirty years. We use techniques from quantum information theory, invariant theory and moment polytopes. We present comparisons among the support functionals and our quantum functionals, and compute generic values. We relate the functionals to instability from geometric invariant theory, in the spirit of Blasiak et al. (Discrete Anal. 2017). We prove that the quantum functionals are asymptotic upper bounds on slice-rank and multi-slice rank, extending a result of Tao and Sawin.
\end{abstract}

\footnotetext{
${ }^{*}$ The full version of this paper is available at https://arxiv.org/abs/1709.07851

Permission to make digital or hard copies of all or part of this work for personal or classroom use is granted without fee provided that copies are not made or distributed for profit or commercial advantage and that copies bear this notice and the full citation on the first page. Copyrights for components of this work owned by others than the author(s) must be honored. Abstracting with credit is permitted. To copy otherwise, or republish, to post on servers or to redistribute to lists, requires prior specific permission and/or a fee. Request permissions from permissions@acm.org.

STOC'18, fune 25-29, 2018, Los Angeles, CA, USA

(C) 2018 Copyright held by the owner/author(s). Publication rights licensed to the Association for Computing Machinery.

ACM ISBN 978-1-4503-5559-9/18/06 . \$ \$15.00

https://doi.org/10.1145/3188745.3188766
}

Furthermore, we make progress on the construction side of the combinatorial version of the asymptotic restriction problem by extending the Coppersmith-Winograd method via combinatorial degeneration. The regular method constructs large free diagonals in powers of any tight set. Our extended version works for any set that has a combinatorial degeneration to a tight set. This generalizes a result of Kleinberg, Sawin and Speyer. As an application we reprove in hindsight recent results on tri-colored sum-free sets by reducing this problem to a result of Strassen on reduced polynomial multiplication.

\section{CCS CONCEPTS}

- Mathematics of computing $\rightarrow$ Information theory; Combinatoric problems; • Theory of computation $\rightarrow$ Quantum information theory; Algebraic complexity theory;

\section{KEYWORDS}

asymptotic restriction, asymptotic spectrum, tensors, fast matrix multiplication, cap set problem, reduced polynomial multiplication, stochastic local operations and classical communication (slocc), entanglement monotones, quantum entropy, moment polytope

ACM Reference Format:

Matthias Christandl, Péter Vrana, and Jeroen Zuiddam. 2018. Universal Points in the Asymptotic Spectrum of Tensors. In Proceedings of 50th Annual ACM SIGACT Symposium on the Theory of Computing (STOC'18). ACM, New York, NY, USA, 8 pages. https://doi.org/10.1145/3188745.3188766

\section{INTRODUCTION}

We study the asymptotic restriction problem, following the pioneering work of Volker Strassen in the sequence of papers [41-44]. This is a mathematical problem with applications in theoretical computer science.

\subsection{The Asymptotic Restriction Problem}

The asymptotic restriction problem is a problem about tensors $t \in \mathbb{F}^{n_{1}} \otimes \cdots \otimes \mathbb{F}^{n_{k}}$ over an arbitrary field $\mathbb{F}$. We need the concepts restriction and tensor product. Let $s \in \mathbb{F}^{n_{1}} \otimes \cdots \otimes \mathbb{F}^{n_{k}}$ and $t \in \mathbb{F}^{m_{1}} \otimes \cdots \otimes \mathbb{F}^{m_{k}}$ be tensors. We say $s$ restricts to $t$, and write $s \geq t$, if there are linear maps $A_{i}: \mathbb{F}^{n_{i}} \rightarrow \mathbb{F}^{m_{i}}$ such that $t=\left(A_{1} \otimes \cdots \otimes A_{k}\right) \cdot s$. The tensor product of $s$ and $t$ is the element $s \otimes t \in \mathbb{F}^{n_{1} m_{1}} \otimes \cdots \otimes \mathbb{F}^{n_{k} m_{k}}$ with coordinates $(s \otimes t)_{i, j}=s_{i} t_{j}$. We say $s$ restricts asymptotically to $t$, written $s \gtrsim t$, if there is a sequence of natural numbers $a(n) \in o(n)$ such that $n+a(n)$ copies of $s$ restrict to $n$ copies of $t$,

$$
s^{\otimes n+a(n)} \geq t^{\otimes n} \quad \text { when } n \rightarrow \infty .
$$


The asymptotic restriction problem is: given $s$ and $t$, decide whether $s \gtrsim t$. We will often be interested in taking either $s$ or $t$ to be a diagonal tensor, and investigate the smallest $\beta$ such that $s^{\otimes \beta n+o(n)} \geq t^{\otimes n}$. We come back to this later and focus on $s \gtrsim t$ for now.

Applications of the asymptotic restriction problem include computing the computational complexity of matrix multiplication in algebraic complexity theory [1, 6, 12, 15-17, 31, 33, 39, 40, 53] (see also $[7,11,30,32])$, deciding the feasibility of an asymptotic transformation between pure quantum states via stochastic local operations and classical communication (slocc) in quantum information theory $[3,19,27,50]$, bounding the size of combinatorial structures like cap sets and tri-colored sum-free sets in additive combinatorics $[2,8,18,20,21,29,46-48]$, and bounding the query complexity of certain properties in algebraic property testing [4, 5, 24, 26, 28, 38]. We will elaborate on these connections later (Section 3 ).

There are naturally two directions in the asymptotic restriction problem, namely finding

(1) obstructions, certificates that prohibit $s \gtrsim t$

(2) constructions, matrices that carry out $s \gtrsim t$.

\subsection{Obstructions}

For obstructions one should think of lower bounds in the sense of computational complexity theory. Strassen introduced in 1986 the theory of asymptotic spectra of tensors [41, 43], which can be viewed as the theory of obstructions in the above sense. A remarkable result of this theory is that the asymptotic restriction problem for a family of tensors $\mathcal{X}$ that is closed under direct sum and tensor product and contains the diagonal tensors $\langle n\rangle$, reduces to finding all maps $\mathcal{X} \rightarrow \mathbb{R}_{\geq 0}$ that are

(a) monotone under restriction $\geq$

(b) multiplicative under tensor product $\otimes$

(c) additive under direct $\operatorname{sum} \oplus$

(d) normalised to have value $n$ at the diagonal tensor $\langle n\rangle$.

Such maps are called spectral points of $\mathcal{X}$. The collection of all these maps is the asymptotic spectrum of $\mathcal{X}$. The direct sum $s \oplus t$ is defined naturally as a tensor in $\left(\mathbb{F}^{n_{1}} \oplus \mathbb{F}^{m_{1}}\right) \otimes \cdots \otimes\left(\mathbb{F}^{n_{k}} \oplus \mathbb{F}^{m_{k}}\right)$ and the diagonal tensor $\langle n\rangle$ for $n \in \mathbb{N}$ is the tensor

$$
\langle n\rangle=\sum_{i=1}^{n} e_{i} \otimes \cdots \otimes e_{i} \in \mathbb{F}^{n} \otimes \cdots \otimes \mathbb{F}^{n}
$$

where $e_{1}, \ldots, e_{n}$ is the standard basis of $\mathbb{F}^{n}$. Properties (a) and (b) are natural properties to obtain an obstruction. Namely, suppose $\xi$ is a map $\mathcal{X} \rightarrow \mathbb{R}_{\geq 0}$ satisfying (a) and (b), and let $s, t \in \mathcal{X}$. If $s \gtrsim t$, then by definition $s^{\otimes n+o(n)} \geq t^{\otimes n}$, and (a) and (b) imply

$$
\xi(s)^{n+o(n)}=\xi\left(s^{\otimes n+o(n)}\right) \geq \xi\left(t^{\otimes n}\right)=\xi(t)^{n},
$$

which implies $\xi(s) \geq \xi(t)$. Turning this around, if $\xi(s)<\xi(t)$ then not $s \gtrsim t$, so $\xi$ yields an obstruction to $s \gtrsim t$. More precisely, Strassen shows in $[41,43]$ that the spectral points are a complete set of monotones for $\gtrsim$.

Theorem 1.1 (Strassen). Let $s, t \in \mathcal{X}$. Then $s \gtrsim t$ if and only if for all spectral points $\xi$ of $\mathcal{X}$ we have $\xi(s) \geq \xi(t)$.

Importantly, Strassen's proof of Theorem 1.1 is nonconstructive and relies on Zorn's lemma.
Given $X$, we are faced with the daunting task of finding all spectral points for $\mathcal{X}$. When $\mathcal{X}$ is taken to be the family of all $k$ tensors these spectral points are called universal. Universal spectral points are particularly useful, as they may serve as obstructions for asymptotic restriction $s \gtrsim t$ for any tensors $s$ and $t$.

To put our results into context, we review the spectral points constructed by Strassen in [44]. We need some notation. For a finite set $X$, let $\mathcal{P}(X)$ be the probability distributions on $X$. For $P \in \mathcal{P}(X)$, let $H(P)$ be the Shannon entropy, $H(P)=-\sum_{x \in X} P(x) \log _{2} P(x)$. For finite sets $I_{1}, \ldots, I_{k}$ and $P \in \mathcal{P}\left(I_{1} \times \cdots \times I_{k}\right)$, let $P_{i} \in \mathcal{P}\left(I_{i}\right)$ be the $i$ th marginal distribution, i.e. $P_{i}(x)=\sum_{a: a_{i}=x} P(a)$. Let $[k]=\{1,2, \ldots, k\}$. Let $C(t)$ be the set of $k$-tuples of ordered bases for $\mathbb{F}^{n_{1}}, \ldots, \mathbb{F}^{n_{k}}$. For $C \in C(t)$, let $\operatorname{supp}_{C} t$ be the support of $t$ with respect to $C$. For $\theta \in \mathcal{P}([k])$ and $t \in \mathbb{F}^{n_{1}} \otimes \cdots \otimes \mathbb{F}^{n_{k}}$, define the maps

$$
\begin{aligned}
& \rho^{\theta}(f)=\min _{C \in C(t)} \max _{P \in \mathcal{P}\left(\operatorname{supp}_{C} t\right)} \sum_{i=1}^{k} \theta(i) H\left(P_{i}\right) \\
& \rho_{\theta}(f)=\max _{C \in C(t)} \max _{P \in \mathcal{P}\left(\max \left(\operatorname{supp}_{C} t\right)\right)} \sum_{i=1}^{k} \theta(i) H\left(P_{i}\right)
\end{aligned}
$$

and define $\zeta^{\theta}(f)=2^{\rho^{\theta}(f)}$ and $\zeta_{\theta}(f)=2^{\rho_{\theta}(f)}$. Here $\max \left(\operatorname{supp}_{C} t\right)$ is the set of maximal points in $\operatorname{supp}_{C} t$ with respect to the product order on $\left[n_{1}\right] \times \cdots \times\left[n_{k}\right]$. The maps $\zeta^{\theta}$ and $\zeta_{\theta}$ are called the upper and lower support functional. Strassen shows the following. Both functionals are $\geq$-monotone and $\langle n\rangle$-normalised. The upper is additive and submultiplicative and the lower is superadditive and supermultiplicative. In general, $\rho_{\theta}(t) \leq \rho^{\theta}(t)$.

Let $S \subseteq[k]$ be nonempty. We may transform $t \in \mathbb{F}^{n_{1}} \otimes \cdots \otimes \mathbb{F}^{n_{k}}$ into a matrix flatten $\{S, \bar{S}\}(t)$ via the natural grouping

$$
\mathbb{F}^{n_{1}} \otimes \cdots \otimes \mathbb{F}^{n_{k}} \rightarrow\left(\bigotimes_{i \in S} \mathbb{F}^{n_{i}}\right) \otimes\left(\bigotimes_{j \notin S} \mathbb{F}^{n_{j}}\right): t \mapsto \operatorname{flatten}_{\{S, \bar{S}\}}(t),
$$

a procedure called flattening. From the properties of matrix rank follows that the map

$$
t \mapsto \operatorname{rk}\left(\operatorname{flatten}_{\{S, \bar{S}\}}(t)\right)
$$

is a universal spectral point. There are $2^{k-1}-1$ such universal spectral points corresponding to the $2^{k-1}-1$ different possible nontrivial flattenings. These maps we call the gauge points. We consider the gauge points as the "trivial" universal spectral points. In fact, $k$ of these gauge points coincide with the upper support functionals $\zeta^{\theta}$ for $\theta \in \mathcal{P}([k])$ supported on a single element of $[k]$.

Tensors whose support in some basis is an antichain are called oblique. These form a strict and nongeneric subfamily of all tensors. Strassen shows that for oblique tensors $\rho_{\theta}(t)=\rho^{\theta}(t)$, in which case he gives a formulation in terms of moment polytopes. So the $\zeta^{\theta}$ are spectral points for the family of oblique tensors. Bürgisser shows in [13] that $\zeta_{\theta}$ is not additive in general and thus not a universal spectral point. It is unknown whether the upper support functional $\zeta^{\theta}$ is a universal spectral point in general.

Before discussing constructions, we mention two important special cases of the asymptotic restriction problem. Namely, applications in theoretical computer science often require that either $s$ or $t$ equals a diagonal tensor $\langle n\rangle=\sum_{i=1}^{n} e_{i} \otimes \cdots \otimes e_{i} \in \mathbb{F}^{n} \otimes \cdots \otimes \mathbb{F}^{n}$. 
Therefore one defines the asymptotic rank of $t$

$$
\underline{\mathrm{R}}(t)=\lim _{n \rightarrow \infty} \mathrm{R}\left(t^{\otimes n}\right)^{1 / n}
$$

and the asymptotic subrank of $t$

$$
\mathrm{Q}(t)=\lim _{n \rightarrow \infty} \mathrm{Q}\left(t^{\otimes n}\right)^{1 / n}
$$

where $\mathrm{R}(t)=\min \{r \in \mathbb{N} \mid t \leq\langle r\rangle\}$ is the tensor rank of $t$ and $\mathrm{Q}(t)=\max \{r \in \mathbb{N} \mid\langle r\rangle \leq t\}$ is the subrank of $t$. From the definitions it follows that every spectral point $\xi$ of $\mathcal{X}$ is an upper bound on asymptotic subrank and a lower bound on asymptotic rank for any tensor $t \in \mathcal{X}$,

and from Theorem 1.1 follows

$$
\underline{Q}(t) \leq \xi(t) \leq \underset{R}{\mathrm{R}}(t),
$$

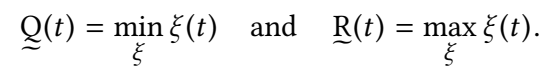

The universal spectral points are upper bounds on asymptotic subrank and lower bounds on asymptotic rank for any tensor. Importantly, for $k \geq 3$, asymptotic subrank $\underline{Q}$ and asymptotic rank $\underset{\sim}{\mathrm{R}}$ are themselves not universal spectral points, since they are not multiplicative under the tensor product $\otimes$.

\subsection{Constructions}

For constructions one should think of fast matrix multiplication algorithms, or large tri-colored sum-free sets. An important construction method is the Coppersmith-Winograd lower bound method as formulated by Strassen in [44] based on a technique in [17].

To state this result we need the concepts tight set, diagonal and free diagonal. Let $\Phi \subseteq I_{1} \times \cdots \times I_{k}$. We say $\Phi$ is tight if there are injective maps $\alpha_{i}: I_{i} \rightarrow \mathbb{Z}_{i}$ such that

$$
\text { if } a \in \Phi \text {, then } \sum_{i=1}^{k} \alpha_{i}\left(a_{i}\right)=0 \text {. }
$$

We call a set $D \subseteq I_{1} \times \cdots \times I_{k}$ a diagonal if any two distinct elements $a, b \in D$ differ in all coordinates, i.e. $\forall i \in[k]: a_{i} \neq b_{i}$. Let $\Phi \subseteq I_{1} \times \cdots \times I_{k}$. We call a diagonal $D \subseteq \Phi$ free for $\Phi$ if

$$
D=\Phi \cap\left(D_{1} \times \cdots \times D_{k}\right),
$$

where $D_{i}=\left\{a_{i} \mid a \in D\right\}$ is the projection of $D$ onto the $i$ th coordinate. We will also say $D \subseteq \Phi$ is a free diagonal. The subrank $\mathrm{Q}(\Phi)$ of $\Phi$ is the largest number $n$ such that there is a free diagonal $D \subseteq \Phi$ of cardinality $|D|=n$. For two sets $\Phi \subseteq I_{1} \times \cdots \times I_{k}$ and $\Psi \subseteq J_{1} \times \cdots \times J_{k}$ we define the product $\Phi \times \Psi \subseteq\left(I_{1} \times J_{1}\right) \times \cdots \times\left(I_{k} \times J_{k}\right)$ by $\Phi \times \Psi=\left\{\left(\left(a_{1}, b_{1}\right), \ldots,\left(a_{k}, b_{k}\right)\right) \mid a \in \Phi, b \in \Psi\right\}$. Note that $\Phi \times \Psi$ again consists of $k$-tuples, and not of $2 k$-tuples. The asymptotic subrank $\mathrm{Q}(\Phi)$ of $\Phi$ is defined as $\mathrm{Q}(\Phi)=\lim _{n \rightarrow \infty} \mathrm{Q}\left(\Phi^{\times n}\right)^{1 / n}$.

We have a notion of subrank for sets $\Phi \subseteq I_{1} \times \cdots \times I_{k}$ and for tensors $t \in \mathbb{F}^{n_{1}} \otimes \cdots \otimes \mathbb{F}^{n_{k}}$ (see Section 1.2). They are related as follows. If $t$ is a tensor with support $\Phi$, then $Q(\Phi) \leq Q(t)$. One may think of the subrank of a set as a stronger notion than the subrank of a tensor, in which local basis transformations are not allowed, just local projections on a subset of the basis.

Strassen proved the following on the asymptotic subrank of a tight set [44, Lemma 5.1]. Let $H_{\theta}(\Phi)=\max _{P \in \mathcal{P}(\Phi)} \sum_{i=1}^{k} \theta(i) H\left(P_{i}\right)$.

Theorem 1.2 (Strassen). Let $\Phi \subseteq I_{1} \times I_{2} \times I_{3}$ be tight. Then

$$
\underline{Q}(\Phi)=\max _{P \in \mathcal{P}(\Phi)} \min _{i \in[3]} 2^{H\left(P_{i}\right)}=\min _{\theta \in \mathcal{P}([3])} 2^{H_{\theta}(\Phi)} \text {. }
$$

The upper bound in (1) is obtained using the support functionals. Note that Theorem 1.2 is about sets of triples only. For a generalisation of the lower bound in (1) to sets of $k$-tuples $\Phi \subseteq I_{1} \times \cdots \times I_{k}$ with $k \geq 4$, see [14].

\section{OUR MAIN RESULTS}

Our main results are (1) the introduction of the first nontrivial universal spectral points, called the quantum functionals, (2) a wide variety of general relationships between our quantum functionals, the Strassen support functionals, slice rank and instability, and (3) an extension to the Coppersmith-Winograd lower bound method via combinatorial degeneration.

\subsection{Introducing Universal Spectral Points beyond the Gauge Points}

We obtain nontrivial spectral points for the family of all complex $k$-tensors, i.e. universal spectral points. This is substantial progress in Strassen's theory of asymptotic spectra, since before this work the only known universal spectral points were the gauge points. (When we say "trivial" universal spectral point we mean gauge point.) We emphasise that these universal spectral points may serve as obstructions for the asymptotic restriction $s \gtrsim t$ of any complex $k$-tensors $s$ and $t$. In particular they serve as upper bounds on asymptotic subrank of any complex $k$-tensor. We stress again that Theorem 1.1 shows the existence of universal spectral points, but its proof is nonconstructive.

To define our universal spectral points we use concepts from quantum information theory such as the quantum entropy function and the partial trace, which we will now introduce. For a general introduction to quantum information theory we refer to the standard reference [52].

Endow the vector space $\mathbb{C}^{d}$ with a Hermitian inner product (one may take the standard Hermitian inner product), so that it is a Hilbert space. A positive semidefinite Hermitian operator $\rho$ : $\mathbb{C}^{d} \rightarrow \mathbb{C}^{d}$ with trace one is called a density operator. The eigenvalues of a density operator $\rho$ form a probability distribution. The quantum entropy or von Neumann entropy $H(\rho)$ is the Shannon entropy of this distribution.

Let $\mathbb{C}^{d_{1}}$ and $\mathbb{C}^{d_{2}}$ be Hilbert spaces. Let $\mathbb{C}^{d_{1}} \otimes \mathbb{C}^{d_{2}}$ be the tensor product Hilbert space. Given a density operator $\rho$ on $\mathbb{C}^{d_{1}} \otimes \mathbb{C}^{d_{2}}$ the reduced density operator $\rho_{1}=\operatorname{tr}_{2} \rho$ is uniquely defined by the property that $\operatorname{tr}\left(\rho_{1} X_{1}\right)=\operatorname{tr}\left(\rho\left(X_{1} \otimes \operatorname{Id}_{d_{2}}\right)\right)$ for all operators $X_{1}$ on $\mathbb{C}^{d_{1}}$. The operator $\rho_{1}$ is again a density operator. The operation $\operatorname{tr}_{2}$ is called the partial trace over $\mathbb{C}^{d_{2}}$. Explicitly, $\rho_{1}$ is given by $\left\langle e_{i}, \rho_{1}\left(e_{j}\right)\right\rangle=\sum_{\ell=1}^{d_{2}}\left\langle e_{i} \otimes e_{\ell}, \rho\left(e_{j} \otimes e_{\ell}\right)\right\rangle$.

Let $\mathbb{C}^{n_{i}}$ be a Hilbert space and let $\mathbb{C}^{n_{1}} \otimes \cdots \otimes \mathbb{C}^{n_{k}}$ be the product Hilbert space. Associate with $t \in \mathbb{C}^{n_{1}} \otimes \cdots \otimes \mathbb{C}^{n_{k}}$ the dual element $t^{*}:=\langle t, \cdot\rangle \in\left(\mathbb{C}^{n_{1}} \otimes \cdots \otimes \mathbb{C}^{n_{k}}\right)^{*}$. Let $\|t\|=\sqrt{\langle t, t\rangle}=1$. Then $t t^{*}=t\langle t, \cdot\rangle$ is a density operator on $\mathbb{C}^{n_{1}} \otimes \cdots \otimes \mathbb{C}^{n_{k}}$. Let $S \subsetneq[k]$ be nonempty. Viewing $t t^{*}$ as a density operator on the regrouped space $\left(\bigotimes_{i \in S} \mathbb{C}^{n_{i}}\right) \otimes\left(\bigotimes_{j \notin S} \mathbb{C}^{n_{j}}\right)$ we may take the partial trace of $t t^{*}$ over $\left(\bigotimes_{i \in S} \mathbb{C}^{n_{i}}\right)$ as described above. We denote the resulting density operator by $\operatorname{Tr}_{S} t$. For $i \in[k]$, we abbreviate $\operatorname{Tr}_{\{i\}}$ to $\operatorname{Tr}_{i}$. 
Let $G$ be the group $\mathrm{GL}\left(\mathbb{C}^{n_{1}}\right) \times \cdots \times \mathrm{GL}\left(\mathbb{C}^{n_{k}}\right)$. For $\theta \in \mathcal{P}([k])$ define the map $F_{\theta}:\{$ complex $k$-tensors $\} \rightarrow \mathbb{R}_{\geq 0}$ by

$$
\begin{aligned}
& F_{\theta}(t)=2^{E_{\theta}(t)} \\
& E_{\theta}(t)=\sup \left\{\sum_{i=1}^{k} \theta(i) H\left(\operatorname{Tr}_{i}(g \cdot t)\right): g \in G ;\|g \cdot t\|=1\right\} .
\end{aligned}
$$

The following is our main result.

Theorem 2.1. $F_{\theta}$ satisfies all four properties (a)-(d) for all complex $k$-tensors.

We outline our approach to proving Theorem 2.1. Let $B$ be the set of bipartitions of $[k]$. Let $\theta \in \mathcal{P}(B)$. We view $[k]$ as a subset of $B$ by mapping $i$ to $\{\{i\},[k] \backslash\{i\}\}$, and thus we view $\mathcal{P}([k])$ as a subset of $\mathcal{P}(B)$. We define the lower quantum functional $F_{\theta}(t)$ as in (3) but with the sum taken over all bipartitions $b \in B$ instead of only the bipartitions of the form $\{i,[k] \backslash\{i\}\}$. That is,

$$
\begin{aligned}
& F_{\theta}(t)=2^{E_{\theta}(t)} \\
& E_{\theta}(t)=\sup \left\{\sum_{b \in B} \theta(b) H\left(\operatorname{Tr}_{b}(g \cdot t)\right): g \in G ;\|g \cdot t\|=1\right\} .
\end{aligned}
$$

For any bipartition $b=\{S, T\}$ of $[k]$ the values of $H\left(\operatorname{Tr}_{S} t\right)$ and $H\left(\operatorname{Tr}_{T} t\right)$ coincide, justifying the notation $\operatorname{Tr}_{b}$.

Next we define the upper quantum functional. Let $V_{1}, \ldots, V_{k}$ be finite-dimensional complex vector spaces. Let $S \subseteq[k]$ be a subset of indices. Define $V_{S}=\otimes_{i \in S} V_{i}$. Under the natural action of the group $\mathrm{GL}\left(V_{S}\right) \times S_{n}$ the space $\left(V_{S}\right)^{\otimes n}$ decomposes as

$$
\left(V_{S}\right)^{\otimes n} \cong \bigoplus_{\lambda r n} \mathbb{S}_{\lambda}\left(V_{S}\right) \otimes[\lambda]
$$

where $[\lambda]$ is an irreducible $S_{n}$-module and $\mathbb{S}_{\lambda}\left(V_{S}\right)$ is an irreducible $\mathrm{GL}\left(V_{S}\right)$-module if $\ell(\lambda) \leq \operatorname{dim} V_{S}$ and 0 if $\ell(\lambda)>\operatorname{dim} V_{S}$. We may then decompose $\left(V_{1} \otimes \cdots \otimes V_{k}\right)^{\otimes n}$ as

$$
\left(V_{1} \otimes \cdots \otimes V_{k}\right)^{\otimes n} \cong\left(V_{S}\right)^{\otimes n} \otimes\left(V_{\bar{S}}\right)^{\otimes n} \cong \bigoplus_{\lambda \vdash n} \mathbb{S}_{\lambda}\left(V_{S}\right) \otimes[\lambda] \otimes\left(V_{\bar{S}}\right)^{\otimes n} .
$$

Let $P_{\lambda}^{V_{S}} \in \operatorname{End}\left(\left(V_{[k]}\right)^{\otimes n}\right)$ be the projection along the above direct sum onto the subspace isomorphic to $\mathbb{S}_{\lambda}\left(V_{S}\right) \otimes[\lambda] \otimes\left(V_{\bar{S}}\right)^{\otimes n}$. For any bipartition $b=\{S,[k] \backslash S\} \in B$, let

$$
P_{\lambda}^{V_{b}}:=P_{\lambda}^{V_{S}} P_{(n)}^{V_{[k]}} \text {. }
$$

This is well-defined, since $P_{\lambda}^{V_{S}} P_{(n)}^{V_{[k]}}=P_{\lambda}^{V_{[k] \backslash S}} P_{(n)}^{V_{[k]}}$. For any partition $\mu=\left(\mu_{1}, \ldots, \mu_{\ell}\right) \vdash n$, let $\bar{\mu}$ be the normalised sequence $\left(\mu_{1} / n, \ldots, \mu_{\ell} / n\right)$. Then $\bar{\mu}$ is a probability distribution. We define the upper quantum functional $F^{\theta}:\{$ complex $k$-tensors $\} \rightarrow \mathbb{R}_{\geq 0}$ by

$$
\begin{aligned}
& F^{\theta}(t)=2^{E^{\theta}(t)} \\
& E^{\theta}(t)=\sup \left\{\sum_{b \in B} \theta(b) H\left(\overline{\lambda^{(b)}}\right):\right. \\
& \left.\quad\left(\lambda^{(b)}\right)_{b \in B} ; \lambda^{(b)} \vdash n ; \prod_{b \in \operatorname{supp} \theta} P_{\lambda^{(b)}}^{V_{b}} t^{\otimes n} \neq 0\right\},
\end{aligned}
$$

i.e. the supremum is over $B$-tuples $\left(\lambda^{(b)}\right)_{b \in B}$ of partitions of $n$ such that $\prod_{b \in \operatorname{supp} \theta} P_{\lambda_{b}(b)}^{V_{b}} t^{\otimes n} \neq 0$.

We say two bipartitions $\{S, T\}$ and $\{X, Y\}$ of $[k]$ are noncrossing if $S \subseteq X$ or $S \subseteq Y$ or $T \subseteq X$ or $T \subseteq Y$. For example, any two bipartitions of the form $\{\{i\},[k] \backslash\{i\}\}$ are noncrossing. First we show the following.

Theorem 2.2. Both $F_{\theta}$ and $F^{\theta}$ are $\geq$-monotone and $\langle n\rangle$-normalised. The map $F_{\theta}$ is $\oplus$-superadditive and $\otimes$-supermultiplicative for every $\theta \in \mathcal{P}(B)$. The $\operatorname{map}^{\theta}(t)$ is $\oplus$-subadditive and $\otimes$-submultiplicative for $\theta \in \mathcal{P}(B)$ supported on mutually noncrossing bipartitions.

To prove Theorem 2.2 we use results on quantum entropy and the semigroup properties of the Kronecker coefficients and LittlewoodRichardson coefficients.

Next we show that $F_{\theta}$ and $F^{\theta}$ coincide when $\theta \in \mathcal{P}([k])$.

Theorem 2.3. $F_{\theta}(t)=F^{\theta}(t)$ when $\theta \in \mathcal{P}([k])$.

Theorem 2.2 and Theorem 2.3 together prove Theorem 2.1. The proof of Theorem 2.3 relies on a connection to entanglement polytopes and a characterisation of these polytopes using equivariant projectors. We give a brief description of the entanglement polytope. Given a tensor $s$, let $r_{i}(s)$ be the ordered spectrum of $\operatorname{Tr}_{i}(s /\|s\|)$. Let $G$ be the group GL $\left(\mathbb{C}^{n_{1}}\right) \times \cdots \times \mathrm{GL}\left(\mathbb{C}^{n_{k}}\right)$. For a tensor $t$, define the set $\Delta_{t}=\left\{\left(r_{1}(s), \ldots, r_{k}(s)\right) \mid s \in \overline{G \cdot t}\right\}$, where $\overline{G \cdot t}$ denotes the Zariski closure of $G \cdot t$. We have

$$
E_{\theta}(t)=\sup \left\{\sum_{i=1}^{k} \theta(i) H\left(P^{(i)}\right) \mid\left(P^{(1)}, \ldots, P^{(k)}\right) \in \Delta_{t}\right\} .
$$

It is a nontrivial fact that $\Delta_{t}$ is a convex polytope $[9,37,51]$. It is called a moment polytope in general or an entanglement polytope in this situation.

\subsection{Comparison of Monotones}

2.2.1 General Relations. First of all, we show that the lower quantum functional is at most the upper quantum functional.

Theorem 2.4. $F_{\theta}(t) \leq F^{\theta}(t)$ when $\theta \in \mathcal{P}(B)$.

For the proof we use the gentle measurement lemma and the quantum estimation theorem from quantum information theory. In the singleton regime $\theta \in \mathcal{P}([k])$ we know that $F_{\theta}(t)=F^{\theta}(t)$ from Theorem 2.3, and we can say even more.

Theorem 2.5. If $\theta \in \mathcal{P}([k])$, then

$$
\begin{array}{r}
\zeta_{\theta}(t) \leq \lim _{n \rightarrow \infty} \zeta_{\theta}\left(t^{\otimes n}\right)^{1 / n} \leq F_{\theta}(t)=F^{\theta}(t)=\lim _{n \rightarrow \infty} \zeta^{\theta}\left(t^{\otimes n}\right)^{1 / n} \\
\leq \zeta^{\theta}(t) .
\end{array}
$$

Finally, we consider free tensors. The concept of free tensors was introduced in [23]. Let $\Phi \subseteq I_{1} \times \cdots \times I_{k}$. We say $\Phi$ is free if the following holds. For any $x, y \in \Phi$, if $x \neq y$, then the tuples $x$ and $y$ differ in at least two coordinates. (This notion of free is not to be confused with the notion of a free diagonal in Section 1.3.) Let $t \in V_{1} \otimes \cdots \otimes V_{k}$. We say $t$ is free if $\operatorname{supp}_{C} t$ is free for some choice of bases $C \in C(t)$. We prove for free tensors that the upper quantum functional and the upper support functional coincide for any $\theta \in \mathcal{P}([k])$.

Theorem 2.6. If $\theta \in \mathcal{P}([k])$ and $t$ is free, then $F^{\theta}(t)=\zeta^{\theta}(t)$.

Our proof of Theorem 2.6 relies on the fact that if a tensor $t$ has a free support $\operatorname{supp}_{C} t$, then the entanglement polytope $\Delta_{t}$ contains for any $P \in \mathcal{P}\left(\operatorname{supp}_{C} t\right)$ the tuple of ordered marginals of $P$. In the special case of $t$ being oblique, Theorem 2.6 corresponds to Corollary 12 in [45]. 
2.2.2 Generic Values. We refer to $\left(n_{1}, \ldots, n_{k}\right)$ as the format of the tensors in $\mathbb{F}^{n_{1}} \otimes \cdots \otimes \mathbb{F}^{n_{k}}$. For $\theta \in \mathcal{P}([k])$ and over appropriate fields, the quantum functionals and support functionals, when restricted to tensors of a specific format, have a generic value, i.e. there is a Zariski open set on which the value of the functional is constant. We denote these generic values by $\zeta^{\theta}\left(n_{1}, \ldots, n_{k}\right), \zeta_{\theta}\left(n_{1}, \ldots, n_{k}\right)$ and $F_{\theta}\left(n_{1}, \ldots, n_{k}\right)$. Clearly,

$$
\begin{aligned}
0 \leq \zeta_{\theta}\left(n_{1}, \ldots, n_{k}\right), \zeta^{\theta}\left(n_{1}, \ldots, n_{k}\right), F_{\theta}\left(n_{1}, \ldots, n_{k}\right) & \\
& \leq n_{1}^{\theta(1)} \cdots n_{k}^{\theta(k)} .
\end{aligned}
$$

Values of $\zeta^{\theta}\left(n_{1}, \ldots, n_{k}\right)$ for comfortable formats were computed by Tobler in [49] and of $\zeta_{\theta}(n, n, n)$ by Bürgisser in [13]. A format $\left(n_{1}, \ldots, n_{k}\right)$ is comfortable if there is a subset $\Phi \subseteq\left[n_{1}\right] \times \cdots \times\left[n_{k}\right]$ and a probability distribution $P \in \mathcal{P}(\Phi)$ such that $\Phi$ is an antichain and each marginal $P_{i}$ is uniform on $\left[n_{i}\right]$. The known values are

(1) $\zeta^{\theta}\left(n_{1}, \ldots, n_{k}\right)=n_{1}^{\theta(1)} \cdots n_{k}^{\theta(k)}$ when $\left(n_{1}, \ldots, n_{k}\right)$ is comfortable

(2) $\zeta_{\theta}(n, n, n)=n^{1-\min _{i} \theta(i)+o(1)}$ when $n \rightarrow \infty$.

We compute $F_{\theta}\left(n_{1}, \ldots, n_{k}\right)$ for formats that allow maximally mixed marginals. We say that $t \in \mathbb{C}^{n_{1}} \otimes \cdots \otimes \mathbb{C}^{n_{k}}$ has maximally mixed marginals if for each $i \in[k]$ the marginal quantum entropy $H\left(\operatorname{Tr}_{i} t\right)$ equals the maximal value $\log _{2} n_{i}$. Bryan et al. in [10] give a characterisation of the formats $\left(n_{1}, \ldots, n_{k}\right)$ for which a tensor with maximally mixed marginals exists. We thank Michael Walter for pointing us to this reference. We have the following.

Theorem 2.7. Let $\theta \in \mathcal{P}([k])$ such that $\theta_{i}>0$ for all $i$. We have

$$
F_{\theta}\left(n_{1}, \ldots, n_{k}\right)=n_{1}^{\theta(1)} \cdots n_{k}^{\theta(k)}
$$

if and only if $\mathbb{C}^{n_{1}} \otimes \cdots \otimes \mathbb{C}^{n_{k}}$ contains a tensor with completely mixed marginals.

Let $t \in \mathbb{C}^{n_{1}} \otimes \cdots \otimes \mathbb{C}^{n_{k}}$. Let $\theta \in \mathcal{P}([k])$. We know from Theorem 2.5 that $E^{\theta}(t) \leq \rho^{\theta}(t) \leq n_{1}^{\theta(1)} \cdots n_{k}^{\theta(k)}$. Therefore, if $E^{\theta}(t)=n_{1}^{\theta(1)} \cdots n_{k}^{\theta(k)}$, then $\rho^{\theta}(t)=n_{1}^{\theta(1)} \cdots n_{k}^{\theta(k)}$. We prove the following converse using the classical Hilbert-Mumford criterion.

Theorem 2.8. Let $t \in \mathbb{C}^{n_{1}} \otimes \cdots \otimes \mathbb{C}^{n_{k}}$. Let $\theta \in \mathcal{P}([k])$ with $\theta_{i}>0$ for all $i$. If $E^{\theta}(t)<n_{1}^{\theta(1)} \cdots n_{k}^{\theta(k)}$, then $\rho^{\theta}(t)<n_{1}^{\theta(1)} \cdots n_{k}^{\theta(k)}$.

2.2.3 Instability. We prove a quantitative version of Theorem 2.8 in terms of instability which moreover holds over all fields. Our result improves a result of Blasiak et al. in [8] when the tensor format $\left(n_{1}, \ldots, n_{k}\right)$ is nonuniform enough.

Instability is a standard notion in geometric invariant theory. Let $\mathbb{F}$ be an algebraically closed field. Let $t \in \mathbb{F}^{n_{1}} \otimes \cdots \otimes \mathbb{F}^{n_{k}}$. Let $G=\mathrm{SL}_{n_{1}}(\mathbb{F}) \times \cdots \times \mathrm{SL}_{n_{k}}(\mathbb{F})$. The tensor $t$ is called unstable if 0 is in the orbit closure $\overline{G \cdot t}$, with the closure taken in the Zariski topology, and otherwise $t$ is called semistable. We use the quantitative notion of instability for a tensor $t \in \mathbb{F}^{n_{1}} \otimes \cdots \otimes \mathbb{F}^{n_{k}}$ defined in [8], with $\mathbb{F}$ an arbitrary field,

$$
\begin{aligned}
\operatorname{instab}(t)= & \max _{C \in C(t)} \max _{w_{1}, \ldots, w_{k}} \sup \left\{\varepsilon \geq 0 \mid \forall a \in \operatorname{supp}_{C} f:\right. \\
& \left.\sum_{i=1}^{k} w_{i}\left(a_{i}\right) \leq \sum_{i=1}^{k}\left(\frac{1}{\left|I_{i}\right|} \sum_{x \in I_{i}} w_{i}(x)-\varepsilon \max _{x} w_{i}(x)\right)\right\}
\end{aligned}
$$

where the first maximum is over the choice of bases of $\mathbb{F}^{n_{1}}, \ldots, \mathbb{F}^{n_{k}}$, with index sets $I_{1}, \ldots, I_{k}$, and the second maximum is over weight functions $w_{i}: I_{i} \rightarrow \mathbb{R}_{\geq 0}$ that are not identically zero. The HilbertMumford criterion says that $t$ is unstable if and only if instab $(t)>0$.

We prove the following upper bound on the support functional using Pinsker's inequality.

Theorem 2.9. Let $t \in \mathbb{F}^{n_{1}} \otimes \cdots \otimes \mathbb{F}^{n_{k}}$. Let $\theta \in \mathcal{P}([k])$. Then

$$
\rho^{\theta}(t) \leq \sum_{i=1}^{k} \theta(i) \log _{2} n_{i}-\frac{2}{\ln 2} \min _{i} \theta(i) \operatorname{instab}(t)^{2} .
$$

2.2.4 Slice Rank. We prove asymptotic upper bounds on slice rank and multi-slice rank in terms of the support functionals and quantum functionals, extending work of Tao and Sawin in [48].

A slice is a tensor of the form $v \otimes w \in V_{1} \otimes \cdots \otimes V_{k}$ where $v \in V_{j}$ and $w \in V_{\overline{\{j\}}}$ for some $j \in[k]$. A multi-slice is a tensor of the form $v \otimes w \in V_{1} \otimes \cdots \otimes V_{k}$ where $v \in V_{S}$ and $w \in V_{\bar{S}}$ for some subset $S \subseteq[k]$. Let $t \in V_{1} \otimes \cdots \otimes V_{k}$. The slice rank of $t$, denoted slicerank $(t)$, is the smallest number $r$ such that $t$ can be written as a sum of $r$ slices. The multi-slice rank of $t$, denoted multislicerank $(t)$, is the smallest number $r$ such that $t$ can be written as a sum of $r$ multi-slices. Both are $\leq$-monotones. The slice rank and multi-slice rank of the diagonal tensor $\langle r\rangle$ equal $r$ [34, 47]. It follows that $\mathrm{Q}(t) \leq$ multislicerank $(t) \leq \operatorname{slicerank}(t)$. Computing upper bounds on subrank $Q(t)$ and asymptotic subrank $Q(t)$ was the motivation for the introduction of slice rank in [47].

Define the asymptotic quantities

$$
\begin{aligned}
\mathrm{SR}^{\sim} & =\limsup _{n \rightarrow \infty} \operatorname{sicerank}\left(t^{\otimes n}\right)^{1 / n} \\
\operatorname{MSR}^{\sim}(t) & =\limsup _{n \rightarrow \infty} \text { multislicerank }\left(t^{\otimes n}\right)^{1 / n} .
\end{aligned}
$$

Then $\underset{\sim}{\mathrm{Q}}(t) \leq \mathrm{MSR}^{\sim}(t) \leq \mathrm{SR}^{\sim}(t)$. We prove the following upper bounds.

Theorem 2.10. Let $t \in \mathbb{C}^{n_{1}} \otimes \cdots \otimes \mathbb{C}^{n_{k}}$. If $\theta \in \mathcal{P}(B)$, then

$$
\operatorname{MSR}^{\sim}(t) \leq F^{\theta}(t) \text {. }
$$

If $\theta \in \mathcal{P}_{\mathrm{s}}(B)$, then

$$
\mathrm{SR}^{\sim}(t) \leq F^{\theta}(t) .
$$

Theorem 2.11. Let $t \in \mathbb{F}^{n_{1}} \otimes \cdots \otimes \mathbb{F}^{n_{k}}$. If $\theta \in \mathcal{P}([k])$, then

$$
\mathrm{SR}^{\sim}(t) \leq \zeta^{\theta}(t) \text {. }
$$

Theorem 2.11 together with Theorem 1.2 show that for tight 3-tensors we have $\mathrm{Q}(t)=\mathrm{SR}^{\sim}(t)$. We remark that slice rank is not equal to subrank (or border subrank) in general, since subrank is super-multiplicative, while slice rank is neither sub-multiplicative nor super-multiplicative. For example, the tensors $\sum_{i=1}^{n} e_{i} \otimes e_{i} \otimes 1$, $\sum_{i=1}^{n} e_{i} \otimes 1 \otimes e_{i}, \sum_{i=1}^{n} 1 \otimes e_{i} \otimes e_{i}$ have slice rank one, while their tensor product equals the matrix multiplication tensor $\langle n, n, n\rangle$ which has slice rank $n^{2}$ (see [8, Remark 4.6]). To see that slice rank is not super-multiplicative, take $W$ to be the tensor $e_{1} \otimes e_{1} \otimes e_{2}+e_{1} \otimes$ $e_{2} \otimes e_{1}+e_{2} \otimes e_{1} \otimes e_{1}$. The slice rank of $W$ equals two. The value of the logarithmic upper support functional $\rho^{(1 / 3,1 / 3,1 / 3)}(W)$ equals $h(1 / 3) \approx 0.918296$. By Theorem 2.11 for any $\theta \in \mathcal{P}([k])$,

$$
\operatorname{slicerank}\left(t^{\otimes n}\right) \leq 2^{\rho^{\theta}(t) n+o(n)}
$$

when $n \rightarrow \infty$. Thus slicerank $\left(W^{\otimes n}\right) \leq 2^{h(1 / 3) n+o(n)}$. 


\subsection{Extending the Coppersmith-Winograd Method}

We extend the Coppersmith-Winograd lower bound method to sets that are not necessarily tight, but have a combinatorial degeneration to a tight set. This has applications in the construction of tri-colored sum-free sets, for example.

Combinatorial degeneration was introduced in [42, Section 6], see also [11, Section 15.6]. Let $\Phi \subseteq \Psi \subseteq I_{1} \times \cdots \times I_{k}$. We say that $\Phi$ is a combinatorial degeneration of $\Psi$, and write $\Psi \unrhd \Phi$, if there are maps $\alpha_{i}: I_{i} \rightarrow \mathbb{Z}(i \in[k])$ such that for $a \in I_{1} \times \cdots \times I_{k}$, if $a \in \Psi \backslash \Phi$, then $\sum_{i=1}^{k} \alpha_{i}\left(a_{i}\right)>0$, and if $a \in \Phi$, then $\sum_{i=1}^{k} \alpha_{i}\left(a_{i}\right)=0$. The definition of combinatorial degeneration is reminiscent of the definition of tightness from Section 1.3. In the definition of combinatorial degeneration, however, the maps $\alpha_{i}$ need not be injective. Our result is the following.

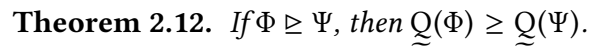

Theorem 2.12 generalizes the technique used by Kleinberg, Sawin and Speyer in [29] to construct large tri-colored sum-free sets, see also Section 3.2.

\section{CONNECTIONS}

We briefly elaborate on the applications of the asymptotic restriction problem stated in the introduction.

\subsection{Matrix Multiplication}

The arithmetic complexity of multiplying two $n \times n$ matrices is characterised by the asymptotic rank of the matrix multiplication tensor

$$
\langle 2,2,2\rangle=\sum_{i, j, k \in[2]} e_{i j} \otimes e_{j k} \otimes e_{k i} \in \mathbb{F}^{2 \times 2} \otimes \mathbb{F}^{2 \times 2} \otimes \mathbb{F}^{2 \times 2} .
$$

In particular, the exponent of matrix multiplication $\omega$ can be expressed in terms of asymptotic rank as

$$
\omega=\log _{2} \underset{\mathrm{R}}{\mathrm{R}}(\langle 2,2,2\rangle),
$$

see e.g. [11]. Strassen in [40] showed that $\omega \leq 2.81$. Currently we know

$$
2 \leq \omega \leq 2.3728639 \text {. }
$$

The upper bound is by Coppersmith and Winograd [17] and improvements by Stothers, Williams and Le Gall [33, 39, 53]. The lower bound follows from a simple flattening argument, or in the asymptotic spectrum framework, from evaluating at a gauge point,

$$
\log _{2} \underset{\sim}{\mathrm{R}}(\langle 2,2,2\rangle) \geq \log _{2} \zeta^{(1,0,0)}(\langle 2,2,2\rangle)=2 .
$$

Our quantum functionals yield the same lower bound $2 \leq \omega$. However, the support functionals and quantum functionals may be useful for ruling out certain approaches to proving $\omega=2$ by giving upper bounds on asymptotic subrank, as we illustrate in Section 3.2.

We finally note that by Theorem 1.1, if the quantum functionals and support functionals are all spectral points for complex 3-tensors, then $\omega=2$.

\subsection{Cap Sets and Tri-colored Sum-free Sets}

We reprove recent results on cap sets and tri-colored sum-free sets using Theorem 2.12 and a result of Strassen on reduced polynomial multiplication. Theorem 2.12 and the support and quantum functionals might be useful in the study of combinatorial objects similar to tri-colored sum-free sets.

We say a subset $A \subseteq(\mathbb{Z} / 3 \mathbb{Z})^{n}$ is a cap set if any line

$$
(u, u+v, u+2 v) \in A^{\times 3}
$$

is a point $(x, x, x)$. We say a subset $M \subseteq\left((\mathbb{Z} / 3 \mathbb{Z})^{n}\right)^{\times 3}$ is a tri-colored sum-free set if $M$ is a free diagonal in

$$
\left\{\left(x_{1}, x_{2}, x_{3}\right) \in\left((\mathbb{Z} / 3 \mathbb{Z})^{n}\right)^{\times 3} \mid x_{1}+x_{2}+x_{3}=0\right\} .
$$

Let $r_{3}\left((\mathbb{Z} / 3 \mathbb{Z})^{n}\right)$ be the size of the largest cap set in $(\mathbb{Z} / 3 \mathbb{Z})^{n}$ and let

$$
r_{3}(\mathbb{Z} / 3 \mathbb{Z})=\lim _{n \rightarrow \infty} r_{3}\left((\mathbb{Z} / 3 \mathbb{Z})^{n}\right)^{1 / n}
$$

Let $s_{3}\left((\mathbb{Z} / 3 \mathbb{Z})^{n}\right)$ be the size of the largest tri-colored sum-free set in $\left((\mathbb{Z} / 3 \mathbb{Z})^{n}\right)^{\times 3}$. Then $r_{3}\left((\mathbb{Z} / m \mathbb{Z})^{n}\right) \leq s_{3}\left((\mathbb{Z} / 3 \mathbb{Z})^{n}\right)$. Let

$$
s_{3}(\mathbb{Z} / 3 \mathbb{Z})=\lim _{n \rightarrow \infty} s_{3}\left((\mathbb{Z} / 3 \mathbb{Z})^{\times n}\right)^{1 / n}
$$

We give a history of bounds on $s_{3}\left((\mathbb{Z} / 3 \mathbb{Z})^{n}\right)$ and $r_{3}\left((\mathbb{Z} / 3 \mathbb{Z})^{n}\right)$. Edel in [20] proved $2.21739 \leq \underline{r}_{3}(\mathbb{Z} / 3 \mathbb{Z})$. Ellenberg and Gijswijt in [21] proved

$$
r_{3}(\mathbb{Z} / 3 \mathbb{Z}) \leq 3(207+33 \sqrt{33})^{1 / 3} / 8 \approx 2.755 .
$$

Blasiak et al. [8] proved that in fact $s_{3}(\mathbb{Z} / 3 \mathbb{Z}) \leq 3(207+33 \sqrt{33})^{1 / 3} / 8$. This upper bound was shown to be an equality in the three papers $[29,35,36]$. The above results were obtained for more general groups, for which we refer to the literature. The EllenbergGijswijt upper bound on $r_{3}(\mathbb{Z} / 3 \mathbb{Z})$ proves the Erdős-Szemerédi sunflower conjecture [22] by a result in [2] and disproves the Coppersmith-Winograd "no three disjoint equivoluminous subsets" conjecture [17], which would have implied $\omega=2$. The Blasiak et al. upper bound on $\underline{r}_{3}(\mathbb{Z} / 3 \mathbb{Z})$ also shows that a certain "simultaneous triple product property" constructions cannot give $\omega=2$ [8].

Strassen in [44] showed using the Coppersmith-Winograd method and the support functionals (Theorem 1.2) that the tight set

$$
\Phi=\left\{\left(x_{1}, x_{2}, x_{3}\right) \in\{0,1,2\}^{\times 3} \mid x_{1}+x_{2}+x_{3}=2\right\},
$$

which is related to reduced polynomial multiplication, i.e. multiplication in the algebra $\mathbb{F}_{3}[x] /\left(x^{3}\right)$, has asymptotic subrank

$$
\mathrm{Q}(\Phi)=H_{(1 / 3,1 / 3,1 / 3)}(\Phi)=3(207+33 \sqrt{33})^{1 / 3} / 8 .
$$

We show the following.

Theorem 3.1. $\underline{s}_{3}(\mathbb{Z} / 3 \mathbb{Z})=\underset{\approx}{Q}(\Phi)$

We emphasise that the significance of our result lies in the explicit connection to the framework of asymptotic spectra and the use of Theorem 2.12, and not in the obtained value.

Proof. Via the relabeling $x_{3} \mapsto x_{3}+2 \bmod 3$, the tri-colored sum-free sets in $(\mathbb{Z} / 3 \mathbb{Z})^{n}$ are precisely the free diagonals in $\Psi^{\times n}$, where

$$
\Psi=\left\{\left(x_{1}, x_{2}, x_{3}\right) \in(\mathbb{Z} / 3 \mathbb{Z})^{\times 3} \mid x_{1}+x_{2}+x_{3}=2\right\} .
$$

So $s_{3}(\mathbb{Z} / 3 \mathbb{Z})=Q(\Psi)$. Observe that there is a combinatorial degener-

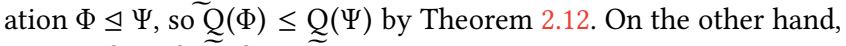
let $t \in \mathbb{F}_{3}^{3} \otimes \mathbb{F}_{3}^{3} \otimes \mathbb{F}_{3}^{3}$ be the tensor with support equal to $\Psi$ and all 
nonzero coefficients equal to one. Then $\mathrm{Q}(\Psi) \leq \underset{Q}{Q}(t)$. Asymptotic subrank is upper bounded by the upper support functional,

$$
\log _{2} \underset{Z}{Q}(t) \leq \min _{C \in C(t)} H_{\theta}\left(\operatorname{supp}_{C} t\right) .
$$

There is a basis such that the support of $t$ equals the set $\Phi$, so $\min _{C \in C(t)} H_{\theta}\left(\operatorname{supp}_{C} t\right) \leq H_{\theta}(\Phi)$, see [8] or the full version of our paper. We thus conclude $Q(\Phi)=\underline{Q}(\Psi)$.

\subsection{Algebraic Property Testing}

We observe that several recent results in property testing are obtained via lower bounds on the asymptotic subrank of a set. We think that the study of property testing might benefit from the asymptotic spectrum viewpoint.

We give triangle-freeness as an example. In general, a property $\mathcal{P}$ is a subset of all functions $\{f: D \rightarrow R\}$ for some domain $D$ and range $R$. For such functions we define the distances $d(f, g)=\mathbb{P}_{x}[f(x) \neq g(x)]$ and $d(f, \mathcal{P})=\min _{g \in \mathcal{P}} d(f, g)$. A onesided $\varepsilon$-tester for $\mathcal{P}$ is an algorithm that makes randomised queries to $f$, and outputs yes with probability 1 for $f \in \mathcal{P}$, and no with probability at least $2 / 3$ if $\operatorname{dist}(f, \mathcal{P}) \geq \varepsilon$. Our task is to determine the query complexity of $\mathcal{P}$, i.e. the minimum number of queries needed by any one-sided $\varepsilon$-tester for $\mathcal{P}$.

A function $f: \mathbb{F}_{2}^{n} \rightarrow \mathbb{F}_{2}$ is triangle-free if there are no $x, y \in \mathbb{F}_{2}^{n}$ such that $f(x)=f(y)=f(x+y)=1$. We observe that formulated in the language of asymptotic spectra, the results of $\mathrm{Fu}$ and Kleinberg in [24] amount to the following statement. The query complexity of triangle-freeness is at least $\Omega\left(1 / \varepsilon^{\alpha}\right)$, where

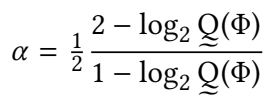

and $\Phi=\{(1,0,0),(0,1,0),(0,0,1)\}$. This bound was generalised to $k$-cycle-freeness in [25].

\section{ACKNOWLEDGMENTS}

The authors thank the members of the QMATH Tensor reading group and Peter Bürgisser for much valuable discussion. Part of this work was carried out while Jeroen Zuiddam and Péter Vrana were visiting QMATH. We acknowledge financial support from the European Research Council (ERC Grant Agreement no. 337603), the Danish Council for Independent Research (Sapere Aude), and VILLUM FONDEN via the QMATH Centre of Excellence (Grant no. 10059). JZ is supported by NWO (617.023.116).

\section{REFERENCES}

[1] Alexander Alder and Volker Strassen. 1981. On the algorithmic complexity of associative algebras. Theoret. Comput. Sci. 15, 2 (1981), $201-211$. https: //doi.org/10.1016/0304-3975(81)90070-0

[2] Noga Alon, Amir Shpilka, and Christopher Umans. 2013. On sunflowers and matrix multiplication. Comput. Complexity 22, 2 (01 Jun 2013), 219-243. https: //doi.org/10.1007/s00037-013-0060-1

[3] Charles H. Bennett, Sandu Popescu, Daniel Rohrlich, John A. Smolin, and Ashish V. Thapliyal. 2000. Exact and asymptotic measures of multipartite pure-state entanglement. Phys. Rev. A 63, 1 (2000), 012307. https://doi.org/10.1103/PhysRevA.63. 012307

[4] Arnab Bhattacharyya, Victor Chen, Madhu Sudan, and Ning Xie. 2010. Testing Linear-Invariant Non-linear Properties: A Short Report. Springer Berlin Heidelberg, Berlin, Heidelberg, 260-268. https://doi.org/10.1007/978-3-642-16367-8 18

[5] Arnab Bhattacharyya and Ning Xie. 2015. Lower bounds for testing trianglefreeness in Boolean functions. Comput. Complexity 24, 1 (01 Mar 2015), 65-101. https://doi.org/10.1007/s00037-014-0092-1
[6] Markus Bläser. 2001. A 5/2 $n^{2}$-Lower Bound for the Multiplicative Complexity of $n \times n$-Matrix Multiplication. STACS 2001 (2001), 99-109. https://doi.org/10. 1007/3-540-44693-1_9

[7] Markus Bläser. 2013. Fast Matrix Multiplication. Number 5 in Graduate Surveys. Theory of Computing Library. 1-60 pages. https://doi.org/10.4086/toc.gs.2013.005

[8] Jonah Blasiak, Thomas Church, Henry Cohn, Joshua A. Grochow, Eric Naslund, William F. Sawin, and Chris Umans. 2017. On cap sets and the group-theoretic approach to matrix multiplication. Discrete Anal. (2017). Issue 3. https://doi.org/ 10.19086/da.1245 arXiv:1605.06702

[9] Michel Brion. 1987. Sur l'image de l'application moment. In Séminaire d'algèbre Paul Dubreil et Marie-Paule Malliavin (Paris, 1986). Lecture Notes in Math., Vol. 1296. Springer, Berlin, 177-192. https://doi.org/10.1007/BFb0078526

[10] Jim Bryan, Zinovy Reichstein, and Mark Van Raamsdonk. 2017. Existence of locally maximally entangled quantum states via geometric invariant theory. arXiv (2017). arXiv: 1708.01645

[11] Peter Bürgisser, Michael Clausen, and M. Amin Shokrollahi. 1997. Algebraic complexity theory. Grundlehren der Mathematischen Wissenschaften, Vol. 315. Springer-Verlag, Berlin. xxiv+618 pages. https://doi.org/10.1007/ 978-3-662-03338-8

[12] Peter Bürgisser and Christian Ikenmeyer. 2011. Geometric Complexity Theory and Tensor Rank. In Proceedings of the Forty-third Annual ACM Symposium on Theory of Computing (STOC '11). ACM, New York, NY, USA, 509-518. https: //doi.org/10.1145/1993636.1993704

[13] Peter Bürgisser. 1990. Degenerationsordnung und Trägerfunktional bilinearer Abbildungen. Ph.D. Dissertation. Universität Konstanz. http://nbn-resolving.de/ urn:nbn:de:bsz:352-opus-20311.

[14] Matthias Christandl, Péter Vrana, and Jeroen Zuiddam. 2016. Asymptotic tensor rank of graph tensors: beyond matrix multiplication. arXiv (2016). arXiv:1609.07476 https://arxiv.org/abs/1609.07476

[15] Henry Cohn, Robert Kleinberg, Balazs Szegedy, and Christopher Umans. 2005. Group-theoretic algorithms for matrix multiplication. In Foundations of Computer Science, 2005. FOCS 2005. 46th Annual IEEE Symposium on. IEEE, 379-388. https: //doi.org/10.1109/SFCS.2005.39

[16] Henry Cohn and Christopher Umans. 2003. A group-theoretic approach to fast matrix multiplication. In Foundations of Computer Science, 2003. Proceedings. 44th Annual IEEE Symposium on. IEEE, 438-449. https://doi.org/10.1109/SFCS.2003. 1238217

[17] Don Coppersmith and Shmuel Winograd. 1990. Matrix multiplication via arithmetic progressions. F. Symbolic Comput. 9, 3 (1990), 251-280. https: //doi.org/10.1016/S0747-7171(08)80013-2

[18] Ernie Croot, Vsevolod F. Lev, and Péter Pál Pach. 2017. Progression-free sets in $\mathbb{Z}_{4}^{n}$ are exponentially small. Ann. of Math. (2) 185, 1 (2017), 331-337. https: //doi.org/10.4007/annals.2017.185.1.7

[19] Wolfgang Dür, Guivre Vidal, and Juan Ignacio Cirac. 2000. Three qubits can be entangled in two inequivalent ways. Phys. Rev. A (3) 62, 6 (2000), 062314, 12. https://doi.org/10.1103/PhysRevA.62.062314

[20] Yves Edel. 2004. Extensions of generalized product caps. Des. Codes Cryptogr. 31, 1 (2004), 5-14. https://doi.org/10.1023/A:1027365901231

[21] Jordan S. Ellenberg and Dion Gijswijt. 2017. On large subsets of $\mathbb{F}_{q}^{n}$ with no three-term arithmetic progression. Ann. of Math. (2) 185, 1 (2017), 339-343. https://doi.org/10.4007/annals.2017.185.1.8

[22] Paul Erdős and Endre Szemerédi. 1978. Combinatorial properties of systems of sets. f. Combinatorial Theory Ser. A 24, 3 (1978), 308-313.

[23] Matthias Franz. 2002. Moment polytopes of projective $G$-varieties and tensor products of symmetric group representations. F. Lie Theory 12, 2 (2002), 539-549. http://emis.ams.org/journals/JLT/vol.12_no.2/16.html

[24] $\mathrm{Hu} \mathrm{Fu}$ and Robert Kleinberg. 2014. Improved Lower Bounds for Testing Triangle-freeness in Boolean Functions via Fast Matrix Multiplication. In Approximation, Randomization, and Combinatorial Optimization. Algorithms and Techniques (APPROX/RANDOM 2014). 669-676. https://doi.org/10.4230/LIPIcs. APPROX-RANDOM.2014.669

[25] Ishay Haviv and Ning Xie. 2015. Sunflowers and testing triangle-freeness of functions. In ITCS'15-Proceedings of the 6th Innovations in Theoretical Computer Science. ACM, New York, 357-366. https://doi.org/10.1145/2688073.2688084 arXiv: 1411.4692

[26] Ishay Haviv and Ning Xie. 2017. Sunflowers and Testing Triangle-Freeness of Functions. Comput. Complexity 26, 2 (01 Jun 2017), 497-530. https://doi.org/10. 1007/s00037-016-0138-7

[27] Ryszard Horodecki, Paweł Horodecki, Michał Horodecki, and Karol Horodecki. 2009. Quantum entanglement. Rev. Modern Phys. 81, 2 (2009), 865-942. https: //doi.org/10.1103/RevModPhys.81.865

[28] Tali Kaufman and Madhu Sudan. 2008. Algebraic Property Testing: The Role of Invariance. In Proceedings of the Fortieth Annual ACM Symposium on Theory of Computing (STOC '08). ACM, New York, NY, USA, 403-412. https://doi.org/10. 1145/1374376.1374434

[29] Robert Kleinberg, William F. Sawin, and David E. Speyer. 2016. The growth rate of tri-colored sum-free sets. arXiv (2016). arXiv:1607.00047 
[30] Joseph M. Landsberg. 2012. Tensors: geometry and applications. Graduate Studies in Mathematics, Vol. 128. American Mathematical Society, Providence, RI. xx+439 pages.

[31] Joseph M. Landsberg. 2014. New lower bounds for the rank of matrix multiplication. SIAM f. Comput. 43, 1 (2014), 144-149. https://doi.org/10.1137/120880276 arXiv: $1206.1530 \mathrm{v} 2$

[32] Joseph M. Landsberg. 2017. Geometry and Complexity Theory. Cambridge University Press.

[33] François Le Gall. 2014. Powers of tensors and fast matrix multiplication. In ISSAC 2014-Proceedings of the 39th International Symposium on Symbolic and Algebraic Computation. ACM, New York, 296-303. https://doi.org/10.1145/2608628.2608664

[34] Eric Naslund. 2017. The multi-slice rank method and polynomial bounds for orthogonal systems in $\mathbb{F}_{q}^{n}$. arXiv (2017). arXiv:1701.04475

[35] Sergey Norin. 2016. A distribution on triples with maximum entropy marginal. arXiv (2016). arXiv: 1608.00243

[36] Luke Pebody. 2016. Proof of a Conjecture of Kleinberg-Sawin-Speyer. arXiv (2016). arXiv:1608.05740

[37] Adam Sawicki, Michał Oszmaniec, and Marek Kuś. 2014. Convexity of momentum map, Morse index, and quantum entanglement. Rev. Math. Phys. 26, 3 (2014), 1450004, 39. https://doi.org/10.1142/S0129055X14500044

[38] Asaf Shapira. 2009. Green's Conjecture and Testing Linear-invariant Properties In Proceedings of the Forty-first Annual ACM Symposium on Theory of Computing (STOC '09). ACM, New York, NY, USA, 159-166. https://doi.org/10.1145/1536414 1536438

[39] Andrew James Stothers. 2010. On the complexity of matrix multiplication. Ph.D. Dissertation. University of Edinburgh. http://hdl.handle.net/1842/4734.

[40] Volker Strassen. 1969. Gaussian elimination is not optimal. Numer. Math. 13, 4 (1969), 354-356. https://doi.org/10.1007/BF02165411

[41] Volker Strassen. 1986. The Asymptotic Spectrum of Tensors and the Exponent of Matrix Multiplication. In Proceedings of the 27th Annual Symposium on Foundations of Computer Science (SFCS '86). IEEE Computer Society, Washington, DC, USA, 49-54. https://doi.org/10.1109/SFCS.1986.52

[42] Volker Strassen. 1987. Relative bilinear complexity and matrix multiplication 7. Reine Angew. Math. 375/376 (1987), 406-443. https://doi.org/10.1515/crll.1987.
375-376.406

[43] Volker Strassen. 1988. The asymptotic spectrum of tensors. F. Reine Angew. Math. 384 (1988), 102-152. https://doi.org/10.1515/crll.1988.384.102

[44] Volker Strassen. 1991. Degeneration and complexity of bilinear maps: some asymptotic spectra. f. Reine Angew. Math. 413 (1991), 127-180. https://doi.org/ 10.1515/crll.1991.413.127

[45] Volker Strassen. 2005. Komplexität und Geometrie bilinearer Abbildungen. Fahresber. Deutsch. Math.-Verein. 107, 1 (2005), 3-31.

[46] Terence Tao. 2008. Structure and randomness: pages from year one of a mathematical blog. American Mathematical Soc.

[47] Terence Tao. 2016. A symmetric formulation of the Croot-Lev-Pach-EllenbergGijswijt capset bound. (2016). https://terrytao.wordpress.com/2016/05/18/ a-symmetric-formulation- of-the-croot-lev-pach-ellenberg-gijswijt-capset-bound https://terrytao.wordpress.com.

[48] Terence Tao and Will Sawin. 2016. Notes on the "slice rank" of tensors. (2016). https://terrytao.wordpress.com/2016/08/24/ notes-on-the-slice-rank-of-tensors/

[49] Verena Tobler. 1991. Spezialisierung und Degeneration von Tensoren. Ph.D. Dissertation. Universität Konstanz. http://nbn-resolving.de/urn:nbn:de:bsz: 352-opus-20324.

[50] F. Verstraete, J. Dehaene, B. De Moor, and H. Verschelde. 2002. Four qubits can be entangled in nine different ways. Phys. Rev. A (3) 65, 5, part A (2002), 052112, 5. https://doi.org/10.1103/PhysRevA.65.052112

[51] Michael Walter, Brent Doran, David Gross, and Matthias Christandl. 2013. Entanglement polytopes: multiparticle entanglement from single-particle information. Science 340, 6137 (2013), 1205-1208. https://doi.org/10.1126/science.1232957 arXiv: 1208.0365

[52] Mark M. Wilde. 2017. Quantum information theory (second ed.). Cambridge University Press, Cambridge. xvii+757 pages. https://doi.org/10.1017/9781316809976

[53] Virginia Vassilevska Williams. 2012. Multiplying matrices faster than Coppersmith-Winograd [extended abstract]. In STOC'12-Proceedings of the 2012 ACM Symposium on Theory of Computing. ACM, New York, 887-898. https://doi.org/10.1145/2213977.2214056 